\title{
A 3-year randomized clinical trial evaluating two different bonded posterior restorations: Amalgam versus resin composite
}

\author{
Hande Kemaloglu ${ }^{1}$, Tijen Pamir ${ }^{1}$, Huseyin Tezel ${ }^{1}$
}

Correspondence: Dr. Hande Kemaloglu

Email: handedalgar@gmail.com
'Department of Restorative Dentistry, Faculty of Dentistry, Ege University, 35100 Bornova, Izmir, Turkiye

\section{ABSTRACT}

Objective: To compare the performance and postoperative sensitivity of a posterior resin composite with that of bonded amalgam in $40(n=20)$ large sized cavities and to evaluate whether resin composite could be an alternative for bonded amalgam. Materials and Methods: This was a randomized clinical trial. Twenty patients in need of at least two posterior restorations were recruited. Authors randomly assigned one half of the restorations to receive bonded amalgam and the other half to composite restorations. Forty bonded amalgams $(n=20)$ and composites $(n=20)$ were evaluated for their performance on modified-US Public Health Service criteria and postoperative sensitivity using visual analogue scale (VAS) for 36-months. Results: Success rate of this study was 100\%. First clinical alterations were rated as Bravo after 1 year in marginal discoloration, marginal adaptation, anatomical form, and surface roughness for both amalgam and composite. At the $3^{\text {rd }}$ year, overall "Bravo" rated restorations were 12 for bonded amalgam and 13 for resin composites. There were no significant differences among the VAS scores of composites and bonded amalgams for all periods $(P>0.05)$ except for the comparisons at the $3^{\text {rd }}$ year evaluation $(P<0.05)$. Conclusions: Within the limitation of this study, both resin composite and bonded amalgam were clinically acceptable. Postoperative sensitivity results tend to decrease more in composite restorations rather than amalgams. Therefore, it was concluded that posterior resin composite can be used even in large sized cavities.

Key words: Bonded amalgam, posterior restoration, postoperative sensitivity, resin composite

\section{INTRODUCTION}

Direct restorations have been commonly used to restore posterior teeth with large and deep cavities due to their equivalent clinical performance to indirect restorations, reduced chairside time, low cost and technique sensitivity. ${ }^{[1,2]}$ Amalgam and resin composites are two frequently used restorative materials that are considered suitable for direct restorations of posterior area. However, American Dental Association (ADA) ${ }^{[3]}$ limits the indications of resin composites to small and moderate sized cavities and United Nations Environment Programme ${ }^{[4]}$ suggests the phase-down use of amalgam due to environmental protection where there is no alternative and suitable restoration material is available. In some developing countries however, there is still ongoing use of amalgam despite of the shortcomings such as lack of adhesion, increased fracture rate in large cavities and debated toxic effects. ${ }^{[5]}$ Furthermore, amalgam needs retentive cavities that reduce the strength of sound tooth. In order to reduce the need of mechanical retention, help to restore tooth integrity, increase fracture resistance and reduce sensitivity by sealing margins, a conservative technique has been introduced for bonding amalgam restorations. ${ }^{[6]}$

Today increasing demand for aesthetic restorations among patients, the crucial concerns on mercury pollution and raised awareness on waste management

This is an open access article distributed under the terms of the Creative Commons Attribution-NonCommercial-ShareAlike 3.0 License, which allows others to remix, tweak, and build upon the work non-commercially, as long as the author is credited and the new creations are licensed under the identical terms.

For reprints contact: reprints@medknow.com

How to cite this article: Kemaloglu H, Pamir T, Tezel H. A 3-year randomized clinical trial evaluating two different bonded posterior restorations: Amalgam versus resin composite. Eur J Dent 2016;10:16-22. DOI: $10.4103 / 1305-7456.175692$ 
of amalgam, has given rise to the development of composite restorations. Their more frequent utilization can also be observed in large cavities for their increased physical properties, ability to conserve tooth structure and easier adaptation techniques that allow placement of larger layers up to $4 \mathrm{~mm} \cdot{ }^{[7,8]}$ However, their long-term survival rate is still controversial because of the extended indications by clinicians for posterior teeth, which originally included only small and medium-sized restorations. In large cavities, the adhesive system preferred may affect the results of clinical success and postoperative sensitivity. In literature studies evaluating the clinical performance of etch-and-rinse adhesives show favorable results. ${ }^{[9]}$ What's more it was advised to use these systems especially in extended cavities where there is still large areas of enamel present. ${ }^{[10]}$ When postoperative sensitivity is of concern the sensitivity scores of etch-and-rinse adhesives were detected to be similar to that of self-etching primer systems. ${ }^{[11]}$

While one can come across several studies that evaluate the clinical performance of direct posterior restorations in the literature there is still a dilemma of selecting suitable posterior restorative material as an amalgam substitute in large cavities. Therefore in this in vivo study, we aimed to evaluate the clinical success and postoperative sensitivity of both bonded amalgam and resin composite restorations after baseline ( 2 weeks), 6 months, 1 and 3 years. The null hypothesis was set to be proven that there is difference in the clinical performance of bonded amalgam and resin composite restorations.

\section{MATERIALS AND METHODS}

This was a randomized clinical trial and the randomization of the restorations was obtained by flipping a coin to choose the first teeth to be restored by the resin composite. Twenty-five patients (aged 18-60) who took part in this study were selected among those who attended the Restorative Clinic of School of Dentistry within the first 3 months of 2010 that are only in need of at least two class II restorations (OD, $\mathrm{MO}$ or mesio-occluso-distal) in molars or premolars. To be included, the teeth to be restored had to: (1) Be asymptomatic (2) have occlusal and adjacent teeth in contact (3) have cavity sizes exceeding the one-third of the faciolingual distance between cusp tips. On the other hand the exclusion criteria were: (1) Patients with fewer than 20 teeth, (2) poor oral hygiene, (3) bruxism, (4) periodontitis, (5) a history of allergic reactions to any of the materials used.
Before participating to this clinical research, the patients were informed about the procedures and signed the consent form which explained the procedures that would be performed during the study. The study was conducted according to the ethical standards stated in the Helsinki Declaration and approved by the Institutional Review Board/Ethics Committee of Ege University.

Each patient received at least two restorations; one with etch-and-rinse adhesive system (XP Bond, Dentsply Caulk, Germany) and posterior resin composite (Quixfil, Dentsply Caulk, Germany) and the second with amalgambond bonding agent (Amalgambond, Parkell Inc., Edgewood NY, USA) and dispersed alloy amalgam (Cavex Holland BV, the Netherlands). Materials used in the study are summarized in Table 1. All restorations were placed by two dentists (H.T. and T.P.) who practiced the technique before they had placed the inaugural restoration. Fifty teeth (molars and premolars) were prepared and restored with bonded amalgam (25) or composite (25) restorations randomly, one of each being in the same patient. The included lesions were placed because of moderately large caries lesions [Figure 1]. One restoration for one appointment was addressed in order to have a reliable sensitivity test.

The operations were performed under local anesthesia if needed. The cavities were performed by using diamond burs under water cooling and the operating fields were isolated with cotton rolls and a suction device.

\section{Resin composite restorations}

Sectional contoured matrix system (Palodent, Dentsply Caulk, Germany) and wooden wedges (Sycamore, Premier Dental Products, Plymouth Meeting, PA. USA), were applied to the cavities which were to be conditioned with $34 \%$ phosphoric acid (Caulk 34\% Tooth Conditioner Gel, Dentsply Caulk, Germany) at a later stage. It was given special emphasis to condition enamel for at least $15 \mathrm{~s}$ and dentin for $15 \mathrm{~s}$ or less, in order to prevent over demineralization. The etch-and-rinse adhesive, (XP Bond, Dentsply Caulk, Germany), was applied to the cavity walls and light-cured for $10 \mathrm{~s}$ with a polymerization light (Elipar Highlight, 3M Espe, Seefeld, Germany) at a minimum intensity of $750 \mathrm{~mW} / \mathrm{cm}^{2}$. Then, posterior resin composite (Quixfil, Dentsply Caulk, Germany) was inserted to the cavities in layers $(4 \mathrm{~mm})$ and light-cured for $20 \mathrm{~s}$. Finishing and polishing of the restorations 
Kemaloglu, et al.: Resin composite as an amalgam substitute

\begin{tabular}{|c|c|c|}
\hline Materials & Type & Composition \\
\hline $\begin{array}{l}\text { DeTrey Conditioner, Dentsply } \\
\text { Caulk, Germany }\end{array}$ & $\begin{array}{l}34 \% \text { tooth } \\
\text { conditioner gel }\end{array}$ & Water, $34 \%$ phosphoric acid, silicon dioxide, surfactants, blue colorant \\
\hline XP Bond, Dentsply Caulk, Germany & $\begin{array}{l}\text { Universal } \\
\text { self-priming } \\
\text { dental adhesive }\end{array}$ & $\begin{array}{l}\text { TCB resin, PENTA, UDMA, TEGDMA, 2-HEMA, butylated benzenediol } \\
\text { (stabilizer), ethyl-4-dimethylaminobenzoate, camphorquinone, } \\
\text { functionalised amorphous silica, t-butanol }\end{array}$ \\
\hline Quixfil, Dentsply Caulk, Germany & $\begin{array}{l}\text { Posterior resin } \\
\text { composite }\end{array}$ & $\begin{array}{l}\text { UDMA, TEGDMA, di- and trimethacrylate resins, carboxylic acid modified } \\
\text { dimethacrylate resin } \\
\text { BHT, UV stabiliser } \\
\text { Camphorquinone, ethyl-4-dimethylaminobenzoate, silanated strontium } \\
\text { aluminum sodium fluoride, phosphate silicate glass }\end{array}$ \\
\hline Enhance, Dentsply Caulk, Germany & Polishing system & Polymerized UDMA resin, aluminum oxide, silicon dioxide \\
\hline $\begin{array}{l}\text { Amalgambond Plus, Parkell, } \\
\text { Farmingdale, USA }\end{array}$ & $\begin{array}{l}\text { Self-cure } \\
\text { bonding system }\end{array}$ & $\begin{array}{l}\text { Activator: } 10 \% \text { citric acid }+3 \% \text { ferric chloride } \\
\text { Adhesive agent: } 35 \% \text { HEMA, water base: } 4-M E T A \text {, MMA, HEMA catalyst: } \\
\text { TBB HPA powder: PMMA }\end{array}$ \\
\hline $\begin{array}{l}\text { Cavex Non Gamma-2 Haarlem } \\
\text { The Netherlands }\end{array}$ & $\begin{array}{l}\text { Dispersed phase } \\
\text { admix amalgam }\end{array}$ & $\begin{array}{l}\text { Alloy powder: Silver } 277.2 \mathrm{mg} \text {, tin } 71.6 \mathrm{mg} \text {, copper } 47.2 \mathrm{mg} \text {, zinc } 4.0 \mathrm{mg} \\
\text { Mercury } 396 \mathrm{mg}\end{array}$ \\
\hline
\end{tabular}

UDMA: Urethane dimethacrylate, TEGDMA: Triethyleneglycol dimethacrylate, BHT: Butylated hydroxyl toluene, HEMA: Hydroxyethyl methacrylate, MMA: Methyl methacrylate, PMMA: Polymethyl methacrylate, TBB: Tributylborane, HPA: High performance additive, UV: Ultraviolet, 4-META: Methacryloxyethyl trimellitate anhydride, TCB: Carboxylic acid modified dimethacrylate, PENTA: Phosphoric acid modified acrylate resin

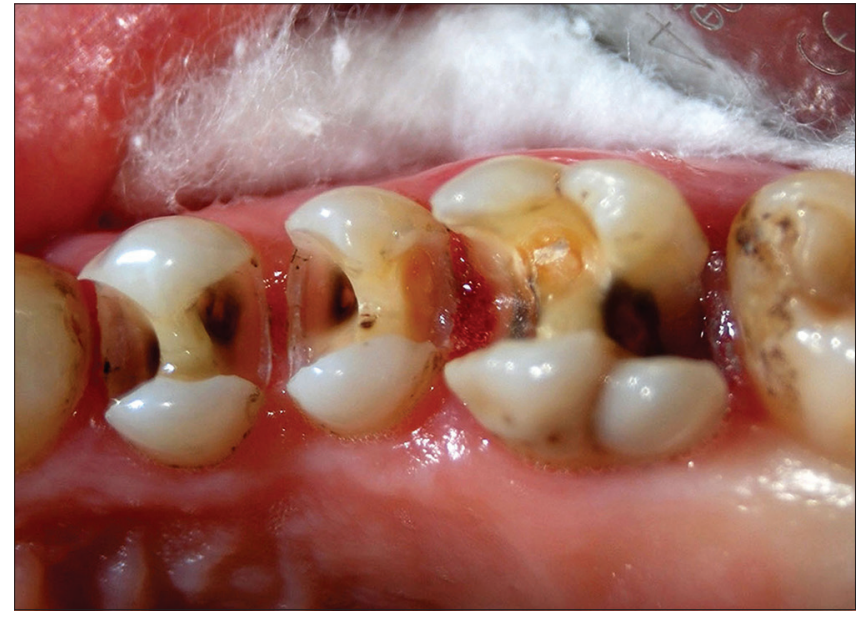

Figure 1: The large sized cavities of this study

were accomplished with fine grit diamond burs, cups and points (Enhance, Dentsply Caulk, Germany).

\section{Amalgam restorations}

Cavities to be restored were limited with metal matrix bands (Hawe Tofflemire, Kerr) and wooden wedges (Sycamore, Premier Dental Products, Plymouth Meeting, PA. USA). The enamel margins and exposed dentinal walls were treated with amalgambond activator for $30 \mathrm{~s}$ and $10 \mathrm{~s}$ respectively. After washing and drying the surface, a thin layer of adhesive agent was brushed onto the activated surfaces followed by the application of base (two drops)/catalyst (one drop) mixture according to manufacturer's instructions. Amalgam had been triturated concurrently with the preparation of amalgambond resin mixture of base/catalyst, thus it has been condensed before the resin mixture dried.
Restorations were finished with fine green stones polished with cups and points after $24 \mathrm{~h}$.

\section{Clinical evaluations}

All restorations were clinically evaluated according to Modified US Public Health Service (USPHS) - Ryge criteria for retention, marginal adaptation, anatomic form, surface texture and secondary caries [Table 2]. In addition to these assessments, postoperative sensitivity levels of each tooth were recorded using visual analogue scale (VAS) in order to transfer patients' subjective feedbacks into objective data. Two examiners who were not involved in the placement of the restorations evaluated the restorations after 2 weeks (baseline), 6 months, 1 and 3 years. In case of disagreement, the examiners reevaluated the restorations until they reached a consensus.

\section{Postoperative sensitivity evaluation}

Postoperative sensitivity evaluation was blindly conducted by a trained examiner after 2 weeks (baseline), 6 months, 1 and 3 years. At each visit, the sensitivity scores were recorded after the application of thermal (cold) stimuli. Thermal stimuli were assessed using a cotton applicator saturated with chloraethyl to the selected tooth. Patients' responses according to VAS have been accepted as the sensitivity value for each tooth and were marked on a VAS, with a $10-\mathrm{cm}$ line labeled from no pain $(0 \mathrm{~cm})$ to intolerable pain $(10 \mathrm{~cm})$. Patients were tried to be examined at the exact scheduled appointment days of the study design. Any changes made in the date of the appointments have been accepted if the related 
date was within minus or plus 1 month $(10 \%$ of the annual appointment time) period.

\section{Statistical analysis}

Based on the Modified Ryge Criteria, we calculated the percentage of Alpha, Bravo, Charlie scores of enrolled bonded amalgam and resin composite restorations. Inter-examiner reliability was evaluated with Cohen's Kappa. Overall failure rate of this study was calculated according to the number of unacceptable restorations using the following formula: ${ }^{[12]}$

Failure percentage $=100 \% \times \frac{\left(\begin{array}{c}\text { previous failures } \\ + \text { new failure }\end{array}\right)}{\left(\begin{array}{c}\text { previous } \\ \text { failures }+ \\ \text { currently recalled } \\ \text { restorations }\end{array}\right)}$

In postoperative sensitivity, the differences between the subject responses were analyzed at baseline, 6 months, 1 and 3 years using the Friedman test followed by Wilcoxon. The significancy was set at $P=0.05$.

\section{RESULTS}

At the beginning there were 25 patients recruited for this randomized in vivo study. However at the end of 2 weeks only 20 patients were available for the recall appointment. We could not establish contact with those five patients who did not complete the study. The examiners evaluated deep and large sized 40 posterior restorations (20 amalgams and 20 resin composites) in 20 patients. Kappa score for inter-examiner agreement for all the evaluation criteria was 0.97 .

The percentages of Alpha ratings for both bonded amalgams and resin composites at baseline, 6 months, 1 and 3 years have been shown in Table 3 [Figure 2a and b]. In this study, retention and secondary caries parameters of each restoration group was $100 \%$. On the other evaluation criteria, clinically acceptable changes started to show up at 1-year recall. On this evaluation period, it was observed that an amalgam and four resin composites caused to marginal discoloration. In addition, two amalgams and two resin composites on marginal adaptation; three amalgams and one resin composite on anatomical form; five amalgams and seven resin composites on surface roughness criteria were rated as "Bravo." At the $3^{\text {rd }}$ year, of all evaluation criteria, overall "Bravo" ratings were 26 for amalgam and 28 for resin composites [Figure 3]. Overall failure rate of this study was $0 \%$ (100\% acceptance for 3 years) which shows that the quality of amalgams and resin composites complied with the requirements for

\begin{tabular}{llll} 
Table 2: Modified USPHS clinical criteria & & \multicolumn{1}{c}{ Score } & Charlie \\
\cline { 2 - 5 } & Alfa & Bravo & Partially or completely lost \\
\hline Retention & Completely retained & Not applicable & Crevice can be detected along the margin \\
Marginal & No crevice can be detected & or base exposed \\
adaptation & along margin by an explorer & but there is no exposure of dentin or base & along margin \\
Anatomical form & Contour of the restoration follows tooth & $\begin{array}{l}\text { Restoration is discontinuous with } \\
\text { tooth, no exposure of dentin or base }\end{array}$ & Restoration has an overhang \\
Marginal & No staining between & Superficial staining along the & Penetration to dentin \\
discoloration & restoration and tooth & margin, no penetration to dentin & Surface of the restoration \\
Surface texture & Surface of the restoration & Surface of the restoration has & has severe defects \\
minimal surface defects & There is clinical diagnosis of caries \\
Secondary caries & There is no clinical diagnosis of caries & Not applicable &
\end{tabular}

\begin{tabular}{|c|c|c|c|c|c|c|c|c|c|c|c|c|c|c|c|c|}
\hline \multirow[t]{3}{*}{ Scores } & \multicolumn{8}{|c|}{ Amalgam } & \multicolumn{8}{|c|}{ Composite } \\
\hline & \multicolumn{2}{|c|}{ Baseline } & \multicolumn{2}{|c|}{6 months } & \multicolumn{2}{|c|}{1 year } & \multicolumn{2}{|c|}{3 years } & \multicolumn{2}{|c|}{ Baseline } & \multicolumn{2}{|c|}{6 months } & \multicolumn{2}{|c|}{1 year } & \multicolumn{2}{|c|}{3 years } \\
\hline & A & B & A & B & A & B & A & B & A & B & A & B & A & B & A & B \\
\hline Retention & 100 & 0 & 100 & 0 & 100 & 0 & 100 & 0 & 100 & 0 & 100 & 0 & 100 & 0 & 100 & 0 \\
\hline Marginal adaptation & 100 & 0 & 100 & 0 & 90 & 10 & 85 & 15 & 100 & 0 & 100 & 0 & 90 & 10 & 80 & 20 \\
\hline Anatomical form & 100 & 0 & 100 & 0 & 85 & 15 & 50 & 50 & 100 & 0 & 100 & 0 & 95 & 5 & 75 & 25 \\
\hline Marginal discoloration & 100 & 0 & 100 & 0 & 95 & 5 & 95 & 5 & 100 & 0 & 100 & 0 & 80 & 20 & 70 & 30 \\
\hline Surface texture & 100 & 0 & 100 & 0 & 75 & 25 & 40 & 60 & 100 & 0 & 100 & 0 & 65 & 35 & 35 & 65 \\
\hline Secondary caries & 100 & 0 & 100 & 0 & 100 & 0 & 100 & 0 & 100 & 0 & 100 & 0 & 100 & 0 & 100 & 0 \\
\hline
\end{tabular}




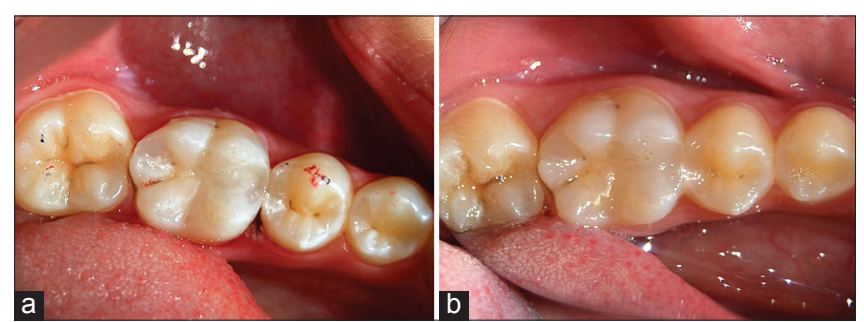

Figure 2: A resin composite restoration scored with Alpha in all evaluation criteria: At baseline (a) and at the end of the third year (b)

minimal standards of Acceptance Program Guideline by ADA. ${ }^{[13]}$

In this study, postoperative sensitivity of the restorations was examined according to patients' responses to cold stimuli using VAS. There were higher number of amalgam restorations exhibiting increased sensitivity at the control periods compared to baseline; however, these increased levels did not present statistical significancy $(P>0.05)$ [Table 4]. For resin composites, postoperative sensitivity tended to decrease generally and variations obtained were significant for all time intervals $(P<0.05)$ [Table 4]. Wilcoxon signed ranks test indicated that there were no significant differences among the VAS scores of resin composites and amalgams for all time periods except for the comparisons at the $3^{\text {rd }}$ year evaluation $(P<0.05)$ [Table 5].

\section{DISCUSSION}

In our study, the clinical success of bonded amalgam and direct resin composite restorations in deep and large sized cavities was evaluated for 3 years. Judging from the results, survival rate was $100 \%$ for both of the restoration types and they were found to be successful.

The type of direct restoration material affects the clinical performance and longevity. Restorations of the small sized cavities with amalgams or resin composites present excellent clinical results due to less amount of material used, reduced cavity margins, easier control of the oral conditions and less chair side time spent. On the other hand, restoration of large sized cavities could include some limitations and challenges when either amalgam or composite were chosen as a direct restoration material. Failure rate of the restorations with four or more surfaces is 4 times greater than that of the single surface restorations due to tooth fractures and secondary caries. ${ }^{[14]}$ As reported in previously published data, secondary caries is the main reason

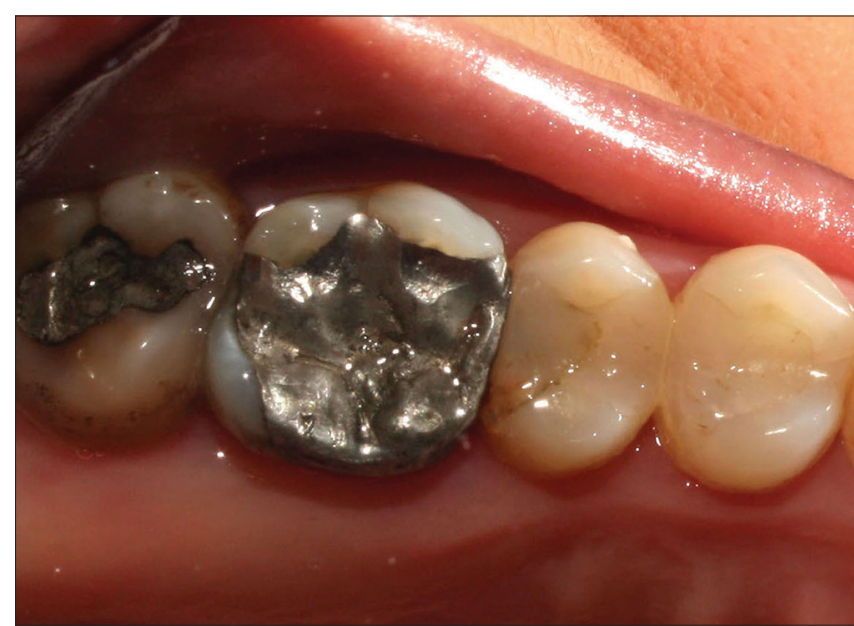

Figure 3: At the end of 3 years, marginal discoloration around the restoration and chipping of the material at palatal area were observed in resin composite of the second premolar. At amalgam restoration besides, failures of anatomical form and surface texture were detected

for failure of both resin composites and amalgam restorations. ${ }^{[15-18]}$ Additionally it was affirmed that evaluation of caries adjacent to composite was faster than amalgam because composite increases the risk for bacterial growth, however in our study, we did not observe any tooth fracture and secondary caries for both types of restorations. Studies concluded that more extensive restorations showed reduced clinical performance, especially the survival rates decrease as the size of restorations increase. ${ }^{[6,19,20]}$ This decline is more noticeable in resin composites than amalgams. In a study, ${ }^{[20]}$ it was reported that after 5 years 14.2 of large amalgam restorations and $19.8 \%$ of large composite restorations had to be replaced. In another study, ${ }^{[15]}$ survival rates of large sized cavities were found to be $89.5 \%$ for amalgam and $74.3 \%$ for composite. Similarly there are several studies showing that amalgam restorations show higher results on longevity, in both small and large cavity size. ${ }^{[21,22]}$ Conversely, in the present study the survival rate of resin composite was found to be $100 \%$ after 3 years of follow-up period. Even though, we did not observe any bulk fracture, chipping was available in one resin restoration of this study. This finding of the present study is in accordance with the conclusion that resin composite can serve adequately even in large sized cavities when they were properly bonded to tooth structure. ${ }^{[23]}$ Therefore, resin composites can undoubtedly be considered as a strong candidate to be used as a substitute for amalgam. Nevertheless, 3 years of evaluation period may not be long enough to make inferences on restorations' inadequacies. The results should be confirmed by the scores that will be obtained from the following recall appointments. 


\begin{tabular}{|c|c|c|c|c|c|c|c|c|c|c|c|c|}
\hline \multirow[t]{3}{*}{ Test groups } & \multicolumn{12}{|c|}{ Variations of VAS scores } \\
\hline & \multicolumn{4}{|c|}{ Baseline - 6 months } & \multicolumn{4}{|c|}{ Baseline - 12 months } & \multicolumn{4}{|c|}{ Baseline - 36 months } \\
\hline & $\begin{array}{l}\text { Negative } \\
\text { ranks }\end{array}$ & $\begin{array}{c}\text { Positive } \\
\text { ranks }\end{array}$ & Ties & Significancy & $\begin{array}{c}\text { Negative } \\
\text { ranks }\end{array}$ & $\begin{array}{l}\text { Positive } \\
\text { ranks }\end{array}$ & Ties & Significancy & $\begin{array}{c}\text { Negative } \\
\text { ranks }\end{array}$ & $\begin{array}{c}\text { Positive } \\
\text { ranks }\end{array}$ & Ties & Significancy \\
\hline Amalgam & 5 & 1 & 14 & $P>0.05$ & 7 & 5 & 8 & $P>0.05$ & 5 & 7 & 8 & $P>0.05$ \\
\hline Resin composite & 7 & 0 & 13 & $P<0.05$ & 9 & 1 & 10 & $P<0.05$ & 10 & 0 & 10 & $P<0.05$ \\
\hline
\end{tabular}

\begin{tabular}{|c|c|c|c|c|}
\hline \multirow{2}{*}{$\begin{array}{l}\text { Resin } \\
\text { composite }\end{array}$} & \multicolumn{4}{|c|}{ Amalgam } \\
\hline & Baseline & $6^{\text {th }}$ month & $12^{\text {th }}$ month $^{\mathrm{c}}$ & $36^{\text {th }}$ month $^{\text {d }}$ \\
\hline Baseline $^{a}$ & $P>0.05$ & & & \\
\hline $6^{\text {th }}$ month $^{\text {b }}$ & & $P>0.05$ & & \\
\hline $12^{\text {th }}$ month $^{\mathrm{b}}$ & & & $P>0.05$ & \\
\hline $36^{\text {th }}$ month $^{\text {b }}$ & & & & $P<0.05$ \\
\hline
\end{tabular}

Different letters indicate statistical significances within each material. Comparisons of the materials according to evaluation periods were presented with $P$ values

Since traditional amalgam restorations did not strengthen tooth structure, fracture was observed often. Therefore, some researchers advocated the use of bonding agents with amalgams declaring that this advantageous procedure reduces early microleakage, fracture, postoperative sensitivity and conserves tooth structure. ${ }^{[24,25]}$ Indeed, we did not observe any failure due to tooth fracture for bonded amalgams. However, we had only one amalgam with small marginal fracture with no exposed base and dentin [Figure 4]. Thus we could repair the restoration easily without the need for replacement of whole restoration. Amalgambond used in this study is a multi-step total etch system that contains 4-meta, a specific monomer. This system is a self-cure adhesive system, which does not need any light curing procedure to set. Amalgam needs to be placed as soon as the bonding agent is applied and condensed in the unset bonding agent. Amalgam particles and the resin sets together forming a locked intermingled impermeable surface. ${ }^{[26]}$ This merged structure may increase the fracture resistance at tooth/restoration unit.

Based on the current developments in material technology, resin composites have started to be used broadly in larger restorations also. In this situation, occlusal wear, polymerization shrinkage, technical complexity of sealing ability, secondary caries risk, failure in determining the margins of the restoration and adaptation of the preferred matrix system are the concerns that still exist. Despite of these concerns, we did not detect any of these mentioned problems except for matrix adaptation. In order to achieve tighter

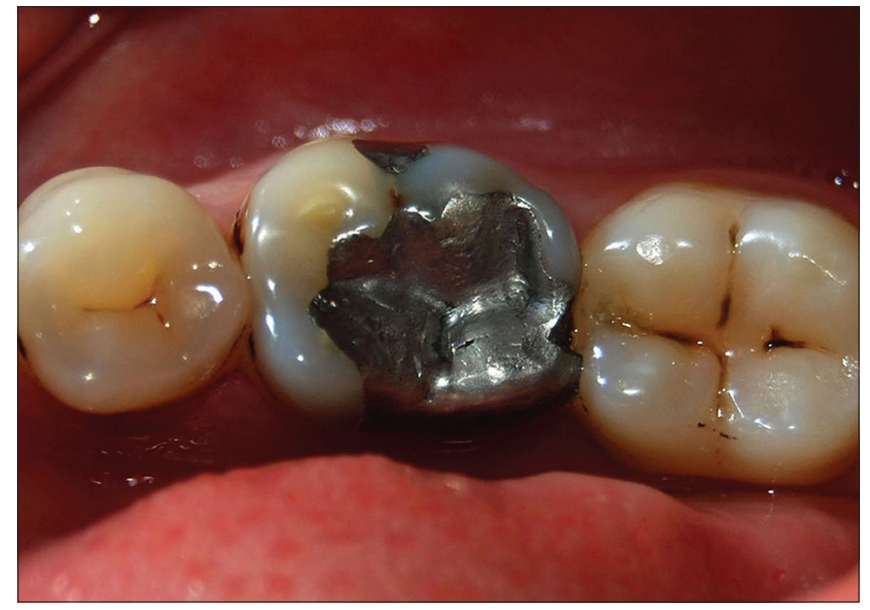

Figure 4: Marginal fracture with no exposed base and dentin in amalgam was rated with Bravo score

proximal contacts in resin composite restorations, dental material manufacturers and researchers advocate using of sectional matrix systems. However, we observed some difficulties in the placement of sectional matrix to cavity lines of large sized cavities resulting in overhang and grooves at gingival area of some restorations.

In the postoperative sensitivity criteria, we found gradual increase in sensitivity levels for bonded amalgam restorations through the end of the investigation period. On the other hand, the sensitivity levels of resin composite restorations decreased throughout the time. These fluctuations observed in sensitivity results were not statistically significant. Furthermore, at the end of the $36^{\text {th }}$ month, sensitivity levels of resin composites were statistically lower than that of the amalgam restorations. Even though both of the restorations were adhesively bonded with etch-and-rinse technique, formulations of etchants, compositions of adhesives and the number of application steps were different from each other. $\mathrm{XP}$ bond used in the resin composites was a one-bottle system with improved ability to diffuse through dentin. When compared to amalgam bond, it has fewer number of application steps, it is less technique sensitive and polymerized with light in $20 \mathrm{~s}$. On the 
contrary, etchant of amalgambond plus contained citric acid and ferric chloride and its priming solvent was acetone. The adhesive systems that contain acetone in their primers require much more attention to form a primed dentin because of their material sensitivity. Therefore, the difference between the postoperative sensitivity of the materials might be attributed to the chemical structure, complex and multi-step procedure of amalgam bond. Furthermore, it was stated that multi-step bonding systems caused gaps in the interface between resin and dentin. These gaps could cause microleakage and postoperative sensitivity. ${ }^{[27]}$ Besides, base-free application might be the reason of elevated postoperative sensitivity of the amalgam restorations due to increased thermal conductivity of the bulk metallic alloys. Therefore, amalgam should be used cautiously when there is risk of postoperative sensitivity.

At the end of 3 years, both bonded amalgam and resin composite were found to be clinically acceptable according to modified USPHS criteria. However, first Bravo ratings for both types of restorations were recorded after 1 year. These minor changes in the quality of the restorations did not need replacement. In postoperative sensitivity criteria, resin composites presented lower sensitivity levels than amalgams after 3 years. Within the limitations of this study, it can be concluded that resin composite can be an alternative for bonded amalgam restorations and can be used with utmost assurance even in large size cavities.

\section{Financial support and sponsorship}

Nil.

\section{Conflicts of interest}

There are no conflicts of interest.

\section{REFERENCES}

1. Brunthaler A, König F, Lucas T, Sperr W, Schedle A. Longevity of direct resin composite restorations in posterior teeth. Clin Oral Investig 2003;7:63-70.

2. Manhart J, Neuerer P, Scheibenbogen-Fuchsbrunner A, Hickel R. Threeyear clinical evaluation of direct and indirect composite restorations in posterior teeth. J Prosthet Dent 2000;84:289-96.

3. Statement on posterior resin-based composites. ADA Council on Scientific Affairs; ADA Council on Dental Benefit Programs. J Am Dent Assoc 1998;129:1627-8.

4. UNEP Dental Amalgam Phase-Down Project East Africa Dental Amalgam Phase-Down (EADAP) Project Promoting the 'Phase Down' Approach of Dental Amalgam in Developing Countries. Available from: http://www.fdiworldental.org/fdi-at-work/programme-forafrica/unep-dental-amalgam-phase-down-project.aspx.[Last accessed on 2015 Dec 30].

5. Opdam NJ, Bronkhorst EM, Loomans BA, Huysmans MC. 12-year survival of composite vs. amalgam restorations. J Dent Res 2010;89:1063-7.

6. Setcos JC, Staninec M, Wilson NH. Bonding of amalgam restorations:
Existing knowledge and future prospects. Oper Dent 2000;25:121-9.

7. Van Ende A, De Munck J, Van Landuyt KL, Poitevin A, Peumans M, Van Meerbeek B. Bulk-filling of high C-factor posterior cavities: Effect on adhesion to cavity-bottom dentin. Dent Mater 2013;29:269-77.

8. Van DijkenJW, Pallesen U. Randomized 3-year clinical evaluation of Class I and II posterior resin restorations placed with a bulk-fill resin composite and a one-step self-etching adhesive. J Adhes Dent 2015;17:81-8.

9. van Dijken JW, Pallesen U. Eight-year randomized clinical evaluation of class II nanohybrid resin composite restorations bonded with a one-step self-etch or a two-step etch-and-rinse adhesive. Clin Oral Investig 2015;19:1371-9.

10. Ozer F, Blatz MB. Self-etch and etch-and-rinse adhesive systems in clinical dentistry. Compend Contin Educ Dent 2013;34:12-4, 16, 18.

11. Perdigão J, Geraldeli S, Hodges JS. Total-etch versus self-etch adhesive: Effect on postoperative sensitivity. J Am Dent Assoc 2003;134:1621-9.

12. Van Dijken JW. A 6-year prospective evaluation of a one-step HEMA-free self-etching adhesive in Class II restorations. Dent Mater 2013;29:1116-22.

13. ADA Council on Scientific Affairs. Direct and indirect restorative materials. J Am Dent Assoc 2003;134:463-72.

14. Soares AC, Cavalheiro A. A review of amalgam and composite longevity of posterior restorations. Rev Port Estomatol Med Dent Cir Maxilofac 2010;51:155-64.

15. Bernardo M, Luis H, Martin MD, Leroux BG, Rue T, Leitão J, et al. Survival and reasons for failure of amalgam versus composite posterior restorations placed in a randomized clinical trial. J Am Dent Assoc 2007; 138:775-83.

16. Manhart J, Chen H, Hamm G, Hickel R. Buonocore memorial lecture. Review of the clinical survival of direct and indirect restorations in posterior teeth of the permanent dentition. Oper Dent 2004;29:481-508.

17. Opdam NJ, Bronkhorst EM, Roeters JM, Loomans BA. A retrospective clinical study on longevity of posterior composite and amalgam restorations. Dent Mater 2007;23:2-8.

18. Celik C, Arhun N, Yamanel K. Clinical evaluation of resin-based composites in posterior restorations: 12-month results. Eur J Dent 2010;4:57-65.

19. Van Nieuwenhuysen JP, D'Hoore W, Carvalho J, Qvist V. Long-term evaluation of extensive restorations in permanent teeth. J Dent 2003;31:395-405.

20. McCracken MS, Gordan VV, Litaker MS, Funkhouser E, Fellows JL, Shamp DG, et al. A 24-month evaluation of amalgam and resin-based composite restorations: Findings from The National Dental Practice-Based Research Network. J Am Dent Assoc 2013;144:583-93.

21. Drummond JL. Degradation, fatigue, and failure of resin dental composite materials. J Dent Res 2008;87:710-9.

22. Soncini JA, Maserejian NN, Trachtenberg F, Tavares M, Hayes C. The longevity of amalgam versus compomer/composite restorations in posterior primary and permanent teeth: Findings From the New England Children's Amalgam Trial. J Am Dent Assoc 2007;138:763-72.

23. Krämer N, García-Godoy F, Reinelt C, Feilzer AJ, Frankenberger R. Nanohybrid vs. fine hybrid composite in extended Class II cavities after six years. Dent Mater 2011;27:455-64.

24. Staninec M, Holt M. Bonding of amalgam to tooth structure: Tensile adhesion and microleakage tests. J Prosthet Dent 1988;59:397-402.

25. Tarim B, Suzuki S, Suzuki S, Cox CF. Marginal integrity of bonded amalgam restorations. Am J Dent 1996;9:72-6.

26. McComb D, Brown J, Forman M. Shear bond strength of resin-mediated amalgam-dentin attachment after cyclic loading. Oper Dent 1995;20:236-40.

27. Opdam NJ, Feilzer AJ, Roeters JJ, Smale I. Class I occlusal composite resin restorations: In vivo post-operative sensitivity, wall adaptation, and microleakage. Am J Dent 1998;11:229-34.

\begin{tabular}{|l|l|}
\hline \multicolumn{2}{|c|}{ Access this article online } \\
\hline Quick Response Code: \\
\hline
\end{tabular}

